\title{
LINFADENECTOMIA AMPLIADA (D2) NO TRATAMENTO DO CARCINOMA GÁSTRICO -ANÁLISE DAS COMPLICAÇÕES PÓS-OPERATÓRIAS
}

\section{EXTENDED LYMPHADENECTOMY (D2) IN THE TREATMENT FOR GASTRIC CANCER - ANALYSIS OF POSTOPERATIVE COMPLICATIONS}

\author{
Marcelo Garcia Toneto, TCBC-RS1; Anselmo Hoffmann²; Antonella Furquim Conte ${ }^{3}$; \\ João Paulo Leal Schambeck ${ }^{3}$; Vinícius Ernani³ ; Hamilton Petry de Souza, TCBC-RS ${ }^{4}$
}

\begin{abstract}
RESUMO: Objetivo: Descrever e analisar as principais complicações pós-operatórias e mortalidade dos pacientes submetidos à ressecção gástrica por câncer gástrico com linfadenectomia D2. Método: Foi realizada uma coorte histórica onde as principais variáveis em estudo foram: idade, localização do tumor, estadiamento, complicações do procedimento cirúrgico, padrão de recidiva tumoral, análise da sobrevida livre de doença e sobrevida total. Resultados: Foram avaliados 35 pacientes submetidos à dissecção linfonodal D2 no período de Janeiro de 2000 a Dezembro de 2004. A média de idade foi 57 anos. Apenas um (2,9\%) paciente apresentava tumor precoce e o local mais comum do tumor foi no terço médio do estômago. O número de linfonodos ressecados por paciente variou de 15 a 80 linfonodos (média 28,8). Vinte e seis $(74,3 \%$ ) pacientes apresentaram linfonodos metastáticos, sendo a média de $13,4( \pm 11,8)$ linfonodos comprometidos por paciente. Seis $(17,1 \%)$ pacientes apresentaram complicações no período pósoperatório, sendo duas pneumonias, uma fístula pancreática, uma fístula do coto duodenal e duas deiscências da anastomose esôfagojejunal. Apenas um $(2,86 \%)$ paciente morreu devido a complicações operatórias. O tempo de seguimento médio foi de 26 meses. Vinte e dois pacientes apresentavam-se vivos no fechamento do estudo, com uma sobrevida atuarial de 62,9\%. Conclusão: Os resultados deste estudo sugerem que, em centros especializados, a linfadenectomia D2 é um procedimento com nível de complicações aceitável e pode ser realizada sem aumento da mortalidade operatória (Rev. Col. Bras. Cir. 2008; 35(4): 228-234).
\end{abstract}

Descritores: Excisão de linfonodo; Gastrectomia; Neoplasias gástricas; Complicações pós-operatórias/mortalidade.

\section{INTRODUÇÃO}

O câncer de estômago é o tumor mais freqüente do trato gastrointestinal e a segunda neoplasia que mais mata em nível global suplantada apenas pelo câncer de pulmão ${ }^{1}$. A ressecção cirúrgica permanece a única opção terapêutica com possibilidade de cura. No entanto, existem temas controversos no manejo desses pacientes, tais como a extensão da ressecção gástrica, a extensão da dissecção linfonodal e a utilização de alternativas terapêuticas adjuvantes (quimio e/ ou radioterapia $)^{2-4}$.

Os avanços nos cuidados pré e pós-operatórios, o desenvolvimento dos antibióticos e o suporte das unidades de tratamento intensivo que ocorreram durante as últimas décadas fizeram com que a mortalidade pós-operatória diminuísse de índices superiores a 50\% para níveis abaixo de $1 \%$ no Japão ${ }^{5}$. Quando são comparados os resultados do tratamento do câncer gástrico no Japão com os resultados obtidos nos Estados Unidos, nota-se nítida melhora no prognóstico nos pacientes orientais ${ }^{6}$. Fator importante nessa avaliação é que o índice de operações em pacientes operados por tumores iniciais é superior a 50\% no Japão contra menos de $10 \%$ nos países ocidentais ${ }^{7}$. Porém, mesmo quando a comparação é realizada entre tumores com o mesmo estadiamento, permanece a vantagem para os pacientes orientais. Algumas hipóteses foram sugeridas por Hundahl ${ }^{8}$ para explicar esses resultados, entre elas: 1 - o fato de que características adquiridas, raciais ou ambientais interfiram na biologia do tumor e na sua relação com o hospedeiro; 2 - a hipótese de que exista uma migração do estágio nos pacientes japoneses, ou seja, como as cirurgias são mais extensas e a avaliação mais meticulosa pelos patologistas, os pacientes seriam mais bem estagiados e avaliados como se fossem de um estágio superior, melhorando os resultados em todos os grupos ${ }^{9} ; 3$ - a possibilidade de melhor tratamento, a qual justificaria o uso dos procedimentos empregados pelos cirurgiões japoneses.

Bonenkamp ${ }^{10}$ e Cushieri ${ }^{11}$, em trabalhos prospectivos randomizados multicêntricos, não conseguiram verificar as vantagens da ressecção ampliada na melhora da sobrevida e ainda demonstraram um aumento do índice de complicações com a ressecção realizada nos moldes da praticada por cirurgiões japoneses. Recentemente, estudo conduzido por

1. Professor Doutor do Departamento de Cirurgia da FAMED-PUCRS

2. Médico Residente do Departamento de Cirurgia da FAMED-PUCRS.

3. Doutorando da FAMED-PUCRS

4. Coordenador Professor Adjunto do Departamento de Cirurgia da FAMED-PUCRS; Chefe do Serviço de Cirurgia Geral e do Aparelho Digestivo do Hospital São Lucas da PUCRS; Fellow do American College of Surgeons; Membro da Associação Brasileira do Câncer Gástrico; Doutor em Cirurgia - UNICAMP.

Recebido em 10/12/2007

Aceito para publicação em 25/03/2008

Conflito de interesses: nenhum

Fonte de financiamento: nenhuma

Trabalho realizado no Departamento de Cirurgia da Faculdade de Medicina da Pontifícia Universidade Católica do Rio Grande do Sul. (FAMEDPUCRS). 
Zilberstein ${ }^{12}$ no Brasil mostrou que a gastrectomia total ou subtotal com linfadenectomia ampliada (D2), realizada de acordo com as recomendações da Sociedade Japonesa para o tratamento do Câncer Gástrico (JRSGC), pode ser considerado um procedimento seguro com mortalidade e morbidade aceitáveis, quando realizado por cirurgiões experientes em centros especializados. Outros trabalhos também demonstram que a linfadenectomia pode ser benéfica para o aumento da sobrevida, desde que a taxa de morbi-mortalidade do procedimento seja baixa ${ }^{13,14}$.

O objetivo deste trabalho é descrever e analisar as principais complicações e mortalidade pós-operatória dos pacientes submetidos à ressecção gástrica por câncer gástrico com linfadenectomia D2, nos padrões recomendados pelos estudos japoneses.

\section{MÉTODO}

Foram estudados todos os pacientes submetidos à gastrectomia com dissecção linfonodal tipo D2 para o tratamento de tumores malignos do estômago por uma equipe especializada em câncer gástrico do Hospital São Lucas da PUCRS, no período de janeiro de 2000 a dezembro de 2004. Após os exames pré-operatórios de estadiamento demonstrarem a inexistência de metástases à distância, os pacientes em condições clínicas de suportar o procedimento foram submetidos a uma videolaparoscopia exploradora. Na ausência de metástases para linfonodos à distância, hepáticas ou implantes peritoneais, gastrectomia com linfadenectomia à D2 foi indicada. Em caso de doença metastática, era indicada operação paliativa, sem dissecção ampliada dos linfonodos. A dissecção linfonodal D2 foi realizada de acordo com as recomendações da JRSSGC ${ }^{15}$. O baço e a cauda do pâncreas foram removidos apenas na evidência de invasão direta pelo tumor.

Os dados obtidos foram analisados a partir de acompanhamento realizado pelos autores no atendimento desses pacientes durante a internação e no período pós-operatório realizado nos ambulatórios de Cirurgia Geral e Cirurgia do Aparelho Digestivo da instituição. Todos os pacientes foram acompanhados até o momento do óbito ou até apresentarem seguimento mínimo de 24 meses após a data do procedimento cirúrgico.

Foram excluídos do estudo todos os casos com tipo histológico que não o adenocarcinoma gástrico, pacientes submetidos a procedimentos cirúrgicos que não se enquadraram na dissecção linfonodal tipo D2, pacientes submetidos a procedimentos paliativos e pacientes submetidos previamente a tratamento rádio e ou quimioterápico.

As principais variáveis em estudo foram: idade, sexo, localização do tumor no estômago, procedimento cirúrgico realizado, estadiamento, classificação de Borrmann e de Lauren, complicações associadas ao procedimento cirúrgico, mortalidade operatória, padrão de recidiva tumoral, análise da sobrevida livre de doença e sobrevida total. O estadiamento utilizado foi a classificação da UICC $2002^{16}$. Quanto ao procedimento cirúrgico, os pacientes foram classificados em três Grupos: gastrectomia parcial, total e esofagogastrectomia. Foi definida como complicação qualquer evento pós-operatório que necessitou algum tipo de tratamento adicional àqueles usualmente utilizados no tratamento cirúrgico para essa do- ença. A mortalidade durante o período pós-operatório foi definida como o óbito nos primeiros 30 dias após a operação, ou até o momento da alta hospitalar ${ }^{17}$.

As variáveis foram caracterizadas por uma análise descritiva, contendo tabelas de médias, com desvio padrão e percentagens. Este estudo foi realizado após aprovação do Comitê de Ética em Pesquisa da Pontifícia Universidade Católica do Rio Grande do Sul.

\section{RESULTADOS}

No período de janeiro de 2000 a dezembro de 2004 , foram incluídos no estudo 35 pacientes operados por adenocarcinoma gástrico com linfadenectomia D2. A idade variou entre 30 e 85 anos, tendo média (dp) $57( \pm 13,8)$ anos. O sexo predominante foi o masculino com $25(71,4 \%)$ pacientes.

O tamanho do tumor variou de 0,7 a $20 \mathrm{~cm}$, média (dp) $6,3( \pm 4,6) \mathrm{cm}$. Os locais mais acometidos foram o terço médio em $14(40 \%)$ casos e o terço distal em 13 (37\%). As principais características anátomo-patológicas dos tumores estão descritas nas tabelas 1 e 2 . Dezessete pacientes $(48,6 \%)$ foram submetidos à gastrectomia total, em $16(45,7 \%)$ foi realizada gastrectomia parcial e dois $(5,7 \%)$ pacientes que apresentavam tumores na cárdia com invasão do esôfago distal foram submetidos à esofagogastrectomia.

O número de linfonodos ressecados por paciente variou entre 15 e 80 linfonodos (média=28,86). Vinte e seis $(74,3 \%)$ pacientes apresentaram linfonodos positivos, sendo a média de $13,4( \pm 11,8)$ linfonodos comprometidos por paciente.

Seis $(17,1 \%)$ pacientes apresentaram complicações no período pós-operatório, sendo duas pneumonias tratadas com antibioticoterapia e suporte respiratório. Um paciente apresentou fístula pancreática após a ressecção da cauda do pâncreas por invasão local da neoplasia, tratada com punção percutânea e octreotide. Um paciente apresentou fístula do coto duodenal tratada com re-operação e suporte nutricional. Dois pacientes apresentaram deiscência da anastomose esôfago-jejunal, sen-

Tabela 1 - Características clínico-patológicas dos pacientes submetidos à gastrectomia com linfadenectomia D2.

\begin{tabular}{lrc}
\hline Característica & N & \% \\
\hline Localização do tumor & & \\
Todo o órgão & 2 & 5,7 \\
Fundo/cárdia & 5 & 14,3 \\
Corpo & 14 & 40 \\
Antro & 13 & 37,1 \\
Coto gástrico & 1 & 2,9 \\
Classificação de Borrmann & & \\
I & 1 & 2,8 \\
II & 10 & 28,5 \\
III & 15 & 42,8 \\
IV & 7 & 20 \\
Classificação de Lauren & & \\
Intestinal & 8 & 22,8 \\
Difuso & 15 & 42,8 \\
Indeterminado & 12 & 34,3 \\
\hline
\end{tabular}


Tabela 2 - Estadiamento ( $p T N M)$.

\begin{tabular}{|c|c|c|c|c|c|}
\hline \multirow{2}{*}{$\frac{\text { Estágio T }}{\mathrm{T} 1}$} & \multicolumn{2}{|c|}{ n $(\%)$} & \multirow{2}{*}{$\frac{\text { Estágio N }}{\text { N0 }}$} & \multicolumn{2}{|c|}{ n $(\%)$} \\
\hline & & $(2,9)$ & & 8 & $(22,9)$ \\
\hline $\mathrm{T} 2$ & & $(17,1)$ & N1 & 12 & $(34,3)$ \\
\hline $\mathrm{T} 3$ & 19 & $(54,3)$ & $\mathrm{N} 2$ & 5 & $(14,3)$ \\
\hline $\mathrm{T} 4$ & 9 & $(25,7)$ & $\mathrm{N} 3$ & 10 & $(28,6)$ \\
\hline Estágio final & \multicolumn{2}{|c|}{ n $(\%)$} & & & \\
\hline IA & & $(5,7)$ & & & \\
\hline IB & & $(11,4)$ & & & \\
\hline II & 3 & $(8,6)$ & & & \\
\hline IIIA & 8 & $(22,9)$ & & & \\
\hline IIIIB & 7 & (20) & & & \\
\hline IV & & $(31,4)$ & & & \\
\hline
\end{tabular}

do que um destes pacientes evoluiu para falência de múltiplos órgãos e óbito, a única mortalidade $(2,86 \%)$ da série.

O tempo de seguimento médio foi de 26 meses, variando entre seis e 72 meses. Vinte e dois pacientes apresentavamse vivos no fechamento do estudo, com sobrevida atuarial de $62,9 \%$. Dos pacientes vivos, que seguem em acompanhamento, oito $(36,4 \%)$ apresentaram recidiva da doença e $14(63,6 \%)$ seguem livres de recidiva tumoral. O principal sítio de recidiva tumoral foi o peritônio em 10 pacientes, seguido pelo fígado em seis, recidiva na anastomose gastro-jejunal em dois e recidiva local com envolvimento do cólon transverso em um paciente.

\section{DISCUSSÃO}

O tratamento recomendado para o adenocarcinoma gástrico é uma ressecção cirúrgica completa, com margens de segurança adequadas em conjunto com a ressecção total do epíploon e dos linfonodos regionais ${ }^{18}$. Na evidência de ascite com presença de células malignas, invasão de estruturas nobres, carcinomatose peritoneal ou doença metastática extensa, o tumor deve ser considerado irressecável e os pacientes somente serão submetidos a procedimentos paliativos para melhora dos sintomas, quando houver obstrução ou sangramento ${ }^{19}$.

A extensão ideal da linfadenectomia vem sendo debatida nas últimas décadas, desde que os cirurgiões japoneses começaram a empregar a linfadenectomia ampliada para o tratamento desta neoplasia com baixo índice de complicações e sobrevida aumentada ${ }^{20}$. Entretanto, os estudos japoneses são criticados por serem séries históricas, não randomizadas e retrospectivas ${ }^{21}$.

Com exceção de centros de excelência no tratamento do câncer gástrico, ${ }^{22,23}$ cirurgiões ocidentais questionam os resultados obtidos no Japão, creditando os melhores resultados ao fenômeno da "migração do estágio". Na busca de evidências científicas para definição do melhor tratamento foram propostos estudos prospectivos, randomizados multicêntricos ${ }^{10,11}$. Porém, a menor incidência desta doença no ocidente gera uma série de dificuldades para realização deste tipo de trabalho. O estudo que alocou maior número de pacientes mostrou que a linfadenectomia D2 aumentava as taxas de complicações e mortalidade, sem melhora na sobrevida. Contudo, foi contestado devido ao grande número de hospitais e cirurgiões recrutados para atingir o núme- ro ideal de pacientes, o que gerou problemas no controle de qualidade das operações. A alta taxa de mortalidade associada ao procedimento neste estudo fez com que ressecções mais limitadas sejam indicadas no mundo ocidental. Entretanto, na análise final do estudo, os autores concluem que as vantagens da linfadenectomia $\mathrm{D} 2$ poderiam ser benéficos se a morbi-mortalidade operatória fosse diminuída ${ }^{24}$. Entretanto, outras vantagens teóricas da linfadenectomia D2 devem ser relatadas, tais como margem mais ampliada de ressecção, estadiamento mais apurado, e a eliminação de possível doença residual nos linfonodos com diminuição da recidiva loco-regional ${ }^{25}$. Além disso, a incidência de metástases nos linfonodos N2 não é desprezível, podendo ocorrer em $25 \%$ dos $\operatorname{casos}^{26}$.

Apenas um paciente desta série apresentava tumor precoce, demonstrando o grau avançado dos tumores tratados nesta instituição ${ }^{27,28}$. Esta é uma variável importante pois o estágio do tumor no momento do diagnóstico é o fator mais importante no prognóstico ${ }^{2}$. Além da importância no prognóstico, o estágio influencia na morbidade, pois lesões mais avançadas tendem a ser tratadas com procedimentos de maior porte, e, portanto, mais sujeitos a complicações. A maioria dos pacientes desta série apresentava tumores em estágio III ou IV, o que demonstra o grau muito avançado da doença no momento do diagnóstico em nosso meio. Deve ser ressaltado que ainda existe um grande atraso na chegada destes doentes para avaliação em centros de referência no Brasil, o que faz com que muitos pacientes não possam ser candidatos ao procedimento cirúrgico adequado por motivo de doença muito avançada, desnutrição e falta de condições clínicas ideais. Um aumento no número de pacientes na amostra deste estudo, principalmente com tumores mais iniciais, contribuiria para definir melhor a relevância da linfadenectomia D2 na mortalidade pós-operatória e sobrevida destes pacientes.

A localização da lesão no órgão é importante, pois define o tipo de ressecção a ser empregado, fator que pode implicar uma maior morbidade e mortalidade associadas ao procedimento $^{28}$. Neste estudo, tumores no terço distal ou tumores do terço médio cuja margem de segurança fosse maior do que $6 \mathrm{~cm}$, foram submetidos à gastrectomia parcial. Nos demais tumores do terço médio e nos localizados no terço proximal, a ressecção foi total. Nos casos de tumores do fun- 
do ou cárdia com invasão do esôfago inferior foi realizada a gastrectomia total com ressecção do esôfago distal.

O número de linfonodos ressecados neste estudo teve ampla variação, apesar da sistemática cirúrgica ser sempre a mesma. A explicação para este achado deve-se ao fato que no início da série a identificação dos linfonodos foi realizada pelo patologista com o material fixado em formalina e, nos casos mais recentes, pelo cirurgião ao final do procedimento com a peça fresca, o que facilita a identificação. A dissecção precisa é fundamental pois estudos prospectivos mostram que o número absoluto de linfonodos ressecados é um método adequado para aferir a qualidade da operação. Karpeh ${ }^{29}$ definiu que 15 seria o número mínimo de linfonodos para estadiamento adequado. Já Siewert ${ }^{30}$ recomenda a ressecção de 25 linfonodos e conclui em análise multivariada que o comprometimento linfonodal é o fator prognóstico independente mais importante para a sobrevida. A relação entre o número de linfonodos metastáticos e ressecados é importante e serve como fator prognóstico independente do tipo de linfadenectomia empregado auxiliando na escolha dos pacientes que possam ser beneficiados pelo tratamento adjuvante.

Marrelli, ${ }^{31}$ analisando as dissecções D2, identificou três situações em que o procedimento está associado a maior risco de complicações: pacientes com pior estado fisiológico, representado por uma classificação ASA mais elevada, necessidade de transfusões sanguíneas e níveis baixos de albumina.

Complicações cardíacas ou pulmonares mais graves não ocorreram nesta série de pacientes, provavelmente devido a seleção dos pacientes. Publicação prévia desta instituição demonstrou que a mortalidade operatória dos pacientes com adenocarcinoma gástrico estava diretamente relacionada às condições fisiológicas dos pacientes e ao estágio do tumor no momento do diagnóstico, independente da técnica cirúrgica empregada ${ }^{28}$. Os pacientes com co-morbidades graves foram submetidos a procedimentos menos invasivos, sem ressecção linfonodal D2.

A principal complicação cirúrgica deste procedimento e um dos maiores responsáveis por óbito nestes pacientes são as deiscências ou fístulas nas linhas de sutura. Quatro pacientes $(11,4 \%)$ apresentaram deiscências ou fístulas, índice semelhante ao encontrado na literatura ${ }^{2,10-12,17,26,31}$. Este deve ser motivo de intensa preocupação do cirurgião no seguimen- to e na busca de atenção a detalhes técnicos. Deiscências de anastomoses e abscessos intra-abdominais são as complicações cirúrgicas mais freqüentes, com até $10 \%$ dos pacientes necessitando re-operações ${ }^{32}$. As deiscências de anastomoses, principalmente a esôfago-jejunal, são a principal causa de mortalidade e ocorrem em 3-10\% dos pacientes ${ }^{33}$. Contudo, com a melhora dos cuidados peri-operatórios, estudos mostram ser possíveis taxas de mortalidade menores que $1 \%{ }^{34}$.

É convicção dos autores que a ausência de fenômenos trombo-embólicos nesta série é devida ao emprego sistemático de medidas de prevenção - uso de meias de compressão, botas de auxílio ao retorno venoso, uso de medicação profilática e saída precoce do leito em todos os pacientes. Todas estas medidas implicaram em tempo de internação hospitalar mais curto, baixa taxa de complicações e índice muito baixo de internação em unidade de tratamento intensivo.

A mortalidade cirúrgica dos pacientes submetidos à ressecção do câncer gástrico varia muito conforme os diversos grupos que tratam dessa doença ${ }^{10,11,25,33,34}$. Os principais fatores de risco para mortalidade operatória são: presença de doenças concomitantes, metástases linfonodais, tamanho do tumor, experiência do grupo de cirurgiões e idade ${ }^{35}$. Zilberstein et al $^{12}$ mostraram que a mortalidade da dissecção linfonodal D2 pode ser cinco vezes menor do que a da dissecção D1 devido a seleção dos pacientes. A baixa mortalidade encontrada nesta série $(2,86 \%)$ provavelmente seja devida à exclusão de operações em tumores muito avançados, pacientes muito idosos e de doentes em más condições de estado fisiológico pré-operatório.

As dificuldades para a realização de trabalhos prospectivos randomizados em cirurgia ${ }^{36}$ devem ser reconhecidas e, alternativas para definir condutas cirúrgicas devem ser intensamente procuradas. Estudos de meta-análise ainda não comprovam os benefícios da D2, e, espera-se que trabalhos bem conduzidos, sem os problemas de curva de aprendizado, taxas elevadas de complicações e controle de qualidade possam definir qual o melhor tratamento para esta neoplasia ${ }^{13}$.

Em conclusão, os resultados deste estudo sugerem que, em centros especializados, a linfadenectomia D2 é um procedimento com nível de complicações aceitável e pode ser realizada sem aumento da mortalidade operatória. Portanto, deve ser incluída em investigações que avaliem a sua efetividade no tratamento cirúrgico do adenocarcinoma gástrico.

\begin{abstract}
Background: The aim of this study was to describe and analyze the postoperative complications and the survival of patients submitted to gastric resection with extended lymphadenectomy. Methods: In a historical cohort, data of patients with gastric carcinoma submitted to D2 lymphadenectomy were studied. The main variables analyzed were: age, tumor location, stage, surgical procedure complications, pattern of tumor recurrence and overall survival. Results: Thirty-five patients were studied during the period between January 2000 and December 2004. Mean age of the patients was 57 years. Only one (2.9\%) patient had early gastric cancer. The most common site was in the middle-third of the stomach. The number of resected nodes per patient ranged from 15 to 80 (mean of 28.8). Twenty-six (74.3\%) patients had metastatic lymph nodes, with mean of $13.4( \pm 11.8)$ positive nodes per patient. Six (17.1\%) patients had complications in the postoperative period, including two pneumonias, one pancreatic fistula, one duodenal stump fistula, and two esophagojejunal leakage. Only one (2.86\%) patient died of operative complications. The meantime of follow-up was 26 months. Twenty-two patients were alive at the conclusion of the study, with a current actuarial survival of $62.9 \%$. Conclusion: The results of this study suggest that, in specialized centers, gastrectomy with D2 lymphadenectomy is a procedure with acceptable levels of complications, and can be performed without increasing the postoperative mortality.
\end{abstract}

Key words: Lymph Node Excision; Gastrectomy; Stomach Neoplasms; Postoperative Complications/mortality. 


\section{REFERÊNCIAS}

1. Parkin DM, Bray FI, Devesa SS. Cancer burden in the year 2000. The global picture. Eur J Cancer. 2001;37(Suppl 8):S466.

2. Brennan MF, Karpeh MS Jr. Surgery for gastric cancer: the American view. Semin Oncol. 1996;23(3):352-9.

3. Macdonald JS, Smalley SR, Benedetti J, Hundahl SA, Estes NC, Stemmermann GN, et al. Chemoradiotherapy after surgery compared with surgery alone for adenocarcinoma of the stomach or gastroesophageal junction. N Engl J Med. 2001;345(10):725-30.

4. Hundahl SA, Macdonald JS, Benedetti J, Fitzsimmons T; Southwest Oncology Group and the Gastric Intergroup. Surgical treatment variation in a prospective, randomized trial of chemoradiotherapy in gastric cancer: the effect of undertreatment. Ann Surg Oncol. 2002;9(3):278-86.

5. Maruyama K, Sasako M, Kinoshita T, Sano T, Katai H. Optimum resection with lymph node dissection for gastric cancer. In: Wanebo HJ, editor. Surgery for gastrointestinal cancer: a multidisciplinary approach. Philadelphia: Lippincott-Raven; 1997. p. 319-27.

6. Wanebo HJ, Kennedy BJ, Winchester DP, Fremgen A, Stewart AK. Gastric carcinoma: does lymph node dissection alter survival? J Am Coll Surg. 1996;183(6):616-24.

7. Nakamura K, Ueyama T, Yao T, Zuan ZX, Ambe K, Adachi Y, et al. Pathology and prognosis of gastric carcinoma. Findings in 10,000 patients who underwent primary gastrectomy. Cancer. 1992;70(5):1030-7.

8. Hundahl SA. Gastric cancer nodal metastasis: biologic significance and therapeutic considerations. Surg Oncol Clin N Am. 1996;5(1):129-44.

9. Bunt AM, Hermans J, Smit VT, van de Velde CJ, Fleuren GJ, Brujin JA. Surgical/pathologic-stage migration confounds comparisons of gastric cancer survival rates between Japan and Western countries. J Clin Oncol. 1995;13(1):19-25.

10. Bonenkamp JJ, Hermans J, Sasako M, van de Velde CJ, Welvaart $\mathrm{K}$, Songun I, et al. Extended lymph-node dissection for gastric cancer. N Eng J Med. 1999;340(12):908-14.

11. Cuschieri A, Fayers P, Fielding J, Craven J, Bancewicz J, Joypaul V, Cook P. Postoperative morbidity and mortality after D1 and D2 resections for gastric cancer: preliminary results of the MRC randomised controlled surgical trial. The Surgical Cooperative Group. Lancet. 1996;347(9007):995-9.

12. Zilberstein B, Martins BC, Jacob CE, Bresciani C, Lopasso FP, Cleva R, et al. Complications of gastrectomy with lymphadenectomy in gastric cancer. Gastric Cancer. 2004;7(4):254-9.

13. McCulloch P, Niita ME, Kazi H, Gama-Rodrigues JJ. Gastrectomy with extended lymphadenectomy for primary treatment of gastric cancer. Br J Surg. 2005;92(1):5-13.

14. Wu CW, Hsiung CA, Lo SS, Hsieh MC, Chen JH, Li AF, Lui WY, Whang-Peng J. Nodal dissection for patients with gastric cancer: a randomised controlled trial. Lancet Oncol. 2006 Apr;7(4):30915. Comment in: Lancet Oncol. 2006 Apr;7(4):279-80. Lancet Oncol. 2006 Jun;7(6):450-1.

15. Nakajima T. Gastric cancer treatment guidelines in Japan. Gastric Cancer. 2002;5(1):1-5.

16. Comparison guide: cancer staging manual. $5^{\text {th }}$ versus $6^{\text {th }}$ ed. Chicago: American Joint Committee on Cancer; 2002 [cited 2007 Apr 4]. Available from: http://www.cancerstaging.org/products/ ajccguide.pdf

17. Schwarz RE, Karpeh MS, Brennan MF. Factors predicting hospitalization after operative treatment for gastric carcinoma in patients older than 70 years. J Am Coll Surg. 1997;184(1):9-15.
18. The Society for Surgery of the Alimentary Tract - SSAT [homepage on the Internet]. Patient care guidelines. Surgical treatment of gastric cancer [cited 2006 Apr 18]. Available from: h t t p://www.ssat.com/cgibin/guidelines _ SurgicalTreatmentGastricCancer_EN.cgi

19. Saito A, Korenaga D, Sakaguchi Y, Ohno S, Ichiyoshi Y, Sugimachi K. Surgical treatment for gastric carcinoma with concomitant hepatic metastasis. Hepatogastroenterology. 1996;43(9):560-4.

20. Maruyama K, Okabayashi K, Kinoshita T. Progress in gastric cancer surgery in Japan and its limits for radicality. World J Surg. 1987;11(4):418-25.

21. Kodera Y, Schwarz RE, Nakao A. Extended lymph node dissection in gastric carcinoma: where do we stand after the Dutch and British randomized trials?

J Am Coll Surg. 2002;195(6):855-64.

22. Scartozzi M, Galizia E, Graziano F, Catalano V, Berardi R, Baldelli $\mathrm{AM}$, et al. Over-DI dissection may question the value of radiotherapy as a part of an adjuvant programme in high-risk radically resected gastric cancer patients. $\mathrm{Br} \mathrm{J}$ Cancer. 2005;92(6):1051-4.

23. Harrison LE, Karpeh MS, Brennan MF. Extended lymphadenectomy is associated with a survival benefit for nodenegative gastric cancer. J Gastrointest Surg. 1998;2(2):126-31.

24. Hartgrink HH, van de Velde CJ, Putter H, Bonenkamp JJ, Kranenbarg EK, Songun I, Welvaart K, et al. Extended lymph node dissection for gastric cancer: who may benefit? Final results of the randomized Dutch gastric cancer group trial. J Clin Oncol. 2004;22(11):2069-77. Epub 2004 Apr 13.

25. Maehara Y, Hasuda S, Koga T, Tokunaga E, Kakeji Y, Sugimachi K. Postoperative outcome and sites of recurrence in patients following curative resection of gastric cancer. Br J Surg. 2000;87(3):353-7.

26. Roviello F, Marrelli D, Morgagni P, de Manzoni G, Di Leo A,Vindigni C, et al; Italian Research Group for Gastric Cancer. Survival benefit of extended D2 lymphadenectomy in gastric cancer with involvement of second level lymph nodes: a longitudinal multicenter study. Ann Surg Oncol. 2002;9(9):894-900.

27. Toneto MG. Análise imunoistoquímica da expressão do fator tecidual e da densidade microvascular no câncer gástrico: correlação com fatores prognósticos e sobrevida [dissertação]. Porto Alegre (RS): Pontifícia Universidade do Rio Grande do Sul; 2006.

28. Toneto MG, Moreira LF, Jeckel Neto E, Souza HP. Gastrectomia em pacientes idosos: análise dos fatores relacionados a complicações e mortalidade. Rev Col Bras Cir. 2004;31(6):373-9.

29. Karpeh MS, Leon L, Klimstra D, Brennan MF. Lymph node staging in gastric cancer: is location more important than Number? An analysis of 1,038 patients. Ann Surg. 2000;232(3):362-71.

30. Siewert JR, Böttcher K, Stein HJ, Roder JD. Relevant prognostic factors in gastric cancer: ten-year results of the German Gastric Cancer Study. Ann Surg. 1998;228(4):449-61.

31. Marrelli D, Pedrazzani C, Neri A, Corso G, DeStefano A, Pinto E, Roviello F. Complications after extended (D2) and superextended (D3) lymphadenectomy for gastric cancer: analysis of potential risk factors Ann Surg Oncol. 2007;14(1): 25-33. Epub 2006 Oct 7.

32. McCulloch P, Ward J, Tekkis PP; ASCOT Group of Surgeons; British Oesophago-Gastroc Cancer Group. Mortality and morbidity in gastro-oesophageal cancer surgery: initial results of ASCOT multicentre prospective cohort study. BMJ. 2003;327(7425):1192-7.

33. Sasako M, Katai H, Sano T, Maruyama K. Management of complications after gastrectomy with extended lymphadenectomy. Surg Oncol. 2000;9(1):31-4. 
34. Park DJ, Lee HJ, Kim HH, Yang HK, Lee KU, Choe KJ. Predictors of operative morbidity and mortality in gastric cancer surgery. Br J Surg. 2005;92(9):1099-102.

35. Siewert JR, Fink U, Sendler A, Becker K, Böttcher K, Feldman HJ, et al. Gastric cancer. Curr Probl Surg. 1997;34(11):835-939. 36. McCulloch P, Taylor I, Sasako M, Lovett B, Griffin D. Randomised trials in surgery: problems and possible solutions. BMJ. 2002 Jun 15;324(7351):1448-51.
Como citar este artigo:

Toneto MG, Hoffmann A, Conte AF, Schambeck JPL, Ernani V, Souza HP. Linfadenectomia alargada (D2) no tratamento do carcinoma gástrico - análise das complicações pós-operatórias e sobrevida. Rev Col Bras Cir. [periódico na Internet] 2008; 35(4). Disponível em URL: http://www.scielo.br/rcbc

Endereço para correspondência: Marcelo Garcia Toneto

Centro Clínico da PUC

Av Ipiranga, 6690 - Conj 612.

90610-000 - Porto Alegre, RS

Fone: 33205179.

E-mail: mtoneto@terra.com.br 